\title{
UNIVERSITY OF KIEL RADIOCARBON MEASUREMENTS VIII*
}

\author{
H ERLENKEUSER, H METZNER, and H WILLKOMM
}

${ }^{14} \mathrm{C}$ Laboratory of the Institut für Reine und Angewandte Kernphysik, University of Kiel

The measurements presented in this list are part of the joint research program "Sonderforschungsbereich 95" of Kiel University for interdisciplinary research on problems of "Interaction Sea-Sea Bottom", sponsored by the Deutsche Forschungsgemeinschaft. They are directed 1) to the study of the path of ${ }^{14} \mathrm{C}$ from the atmosphere into argillaceous sediments, and 2) to date stratigraphic records within sediment. Associated problems are to identify sources of different ${ }^{14} \mathrm{C}$ activity that contribute to recent deposits, and to learn about effects of morphologic and hydrographic factors, especially of bottom currents, on sedimentation sequence. Even short term processes can be successfully studied by analyses of bomb produced radiocarbon in the marine environment.

Results show that biogenic carbon, in the organic part as well as in the carbonate, is in isotopic equilibrium with water environment, which is valid even for benthic communities of muddy sediments in the deeper parts of Kiel Bight. Though most of the organic fraction in the sediments is of autochthonous origin a noticeable amount of carbon with different ${ }^{14} \mathrm{C}$ activity is supplied from other sources. Anthropogenic effects are particularly prevalent in the top layers of the sediment. Reduced sedimentation rates and erosional events are recognized in the sediments of the Kiel Bight channels.

According to the recommendations of the latest ${ }^{14} \mathrm{C}$ conference (Rafter \& Grant-Taylor, 1972), ages are calculated with the Libby halflife, and refer to $95 \%$ of the NBS oxalic acid activity. ${ }^{14} \mathrm{C}$ activities figures listed for modern samples refer to that same standard activity. Data are not corrected for isotopic effects. Errors correspond to 1 standard deviation of the net counting rate including statistics of background and reference standard. $\delta^{13} \mathrm{C}$ values with suffix $\mathrm{S}$ refer to an internal standard of Solnhofen limestone, which is not yet connected to the PDB scale. They may be converted approximately by adding a correction term of $-1 \%$.

Pretreatment procedures and $\mathrm{CO}_{2}$ purification techniques are the same as reported in Willkomm \& Erlenkeuser (1970). However, wet oxidation by means of potassium bichromate and sulfuric acid according to $3 \mathrm{C}+2 \mathrm{~K}_{2} \mathrm{Cr}_{2} \mathrm{O}_{7}+8 \mathrm{H}_{2} \mathrm{SO}_{4} \rightarrow 3 \mathrm{CO}_{2}+2 \mathrm{Cr}_{2}\left(\mathrm{SO}_{4}\right)_{3}+2 \mathrm{~K}_{2} \mathrm{SO}_{4}+8 \mathrm{H}_{2} \mathrm{O}$ resulted in markedly lower gas impurities, especially with sediment samples. The conversion yield was studied on different materials (coal, graphite, charcoal, wood, sediments), and quantitative conversion (better than $97 \%$ ) was found in all cases. The programs for the evaluation of ${ }^{14} \mathrm{C}$-count rates (Erlenkeuser \& Willkomm, 1972, 1973a) were modified for

* Contribution No. 11 of the Sonderforschungsbereich 95 "Interaction Sea-Sea Bottom", University of Kiel. 
a PDP 10 timesharing computer, for greater efficiency in processing the ${ }^{14} \mathrm{C}$ measurements.

\section{ACKNOWLEDGMENTS}

We are indebted to many colleagues of the Geologisch-Paläontologisches Inst, Universität Kiel and of the Inst $\mathrm{f}$ Meereskunde, Universität Kiel for helpful discussions and assistance in collecting samples, especially E Suess, Chr Samtleben, H J Black, and R R Dries. We particularly wish to thank F Werner for supplying sediment cores from Kiel Bight, G Unsöld for collecting mud samples from Nordstrand I., and W Richter for exposing floating plates. We thank V Lohr for processing the Cyprina series. Investigations were financially supported by the Deutsche Forschungsgemeinschaft within the Sonderforschungsbereich 95. The data processing in ${ }^{14} \mathrm{C}$ measurements was performed at the Computer Center of the University of Kiel.

\section{A. Sea water samples}

\section{Kiel Bight series, 1972-1974}

Water samples were coll, in monthly intervals, mainly from Vejsnäs channel, N Kiel Bight, and from Boknis channel, W Kiel Bight. 50L samples were taken from the water column at 5 to $7 \mathrm{~m}$ intervals with a submergible pump and coll in $25 \mathrm{~L}$ plastic bottles. Bacterial activity was ended by adding $\mathrm{I}_{2}-\mathrm{KI}$-solution (Mook, 1970). $\mathrm{CO}_{2}$ was released at $\mathrm{pH}=$ 2 at room temperature, flushed by a stream of nitrogen bubbled through sample for ca $8 \mathrm{hr}$ at $0.6 \mathrm{~L} \mathrm{NTP} / \mathrm{min}$, dried in concentric sulfuric acid and trapped at $-194^{\circ} \mathrm{C}$ while carrier gas was fed back to sample. Results appear in Table 1.

General Comment: Kiel Bight is characterized by brackish water with strong variations of hydrographic parameters. High salinity water originating from the Kattegat Sea in the $\mathrm{N}$ enters Kiel Bight through Great Belt channel and is distributed at the base via Vejsnäs and Boknis channel into W and SW Kiel Bight (Wattenberg, 1949). The surface layer, however, is affected by outflow of low salinity water from the Central Baltic. Most of the year, Kiel Bight water shows pronounced saline stratification with salinity ca 22\%o (seasonal range: 14 to $30 \%$ ) in the basal layer below 20m water depth, and ca 15\%o (seasonal range: 9 to 20\%o) in the surface layer above 10m (for details, see Wattenberg, 1949; Seibold et al, 1971). ${ }^{14} \mathrm{C}$ activity is high in the Baltic Sea (see Bornholm series) due to $\mathrm{CO}_{2}$ exchange with the atmosphere, while it is kept low in the Kattegat Sea indicating combined influence of the Norwegian and North Seas (for ${ }^{14} \mathrm{C}$ level, see Gulliksen \& Nydal, 1972). ${ }^{14} \mathrm{C}$ level in Kiel Bight water is intermediate and is correlated with salinity. With strong bottom water inflow, eg, July, 1972, however, ${ }^{14} \mathrm{C}$ activity is lower than calculated from a 2-box mixing model. This might indicate a stronger supply of low activity waters from the Norwegian Sea via the deep channels in the Kattegat area. 


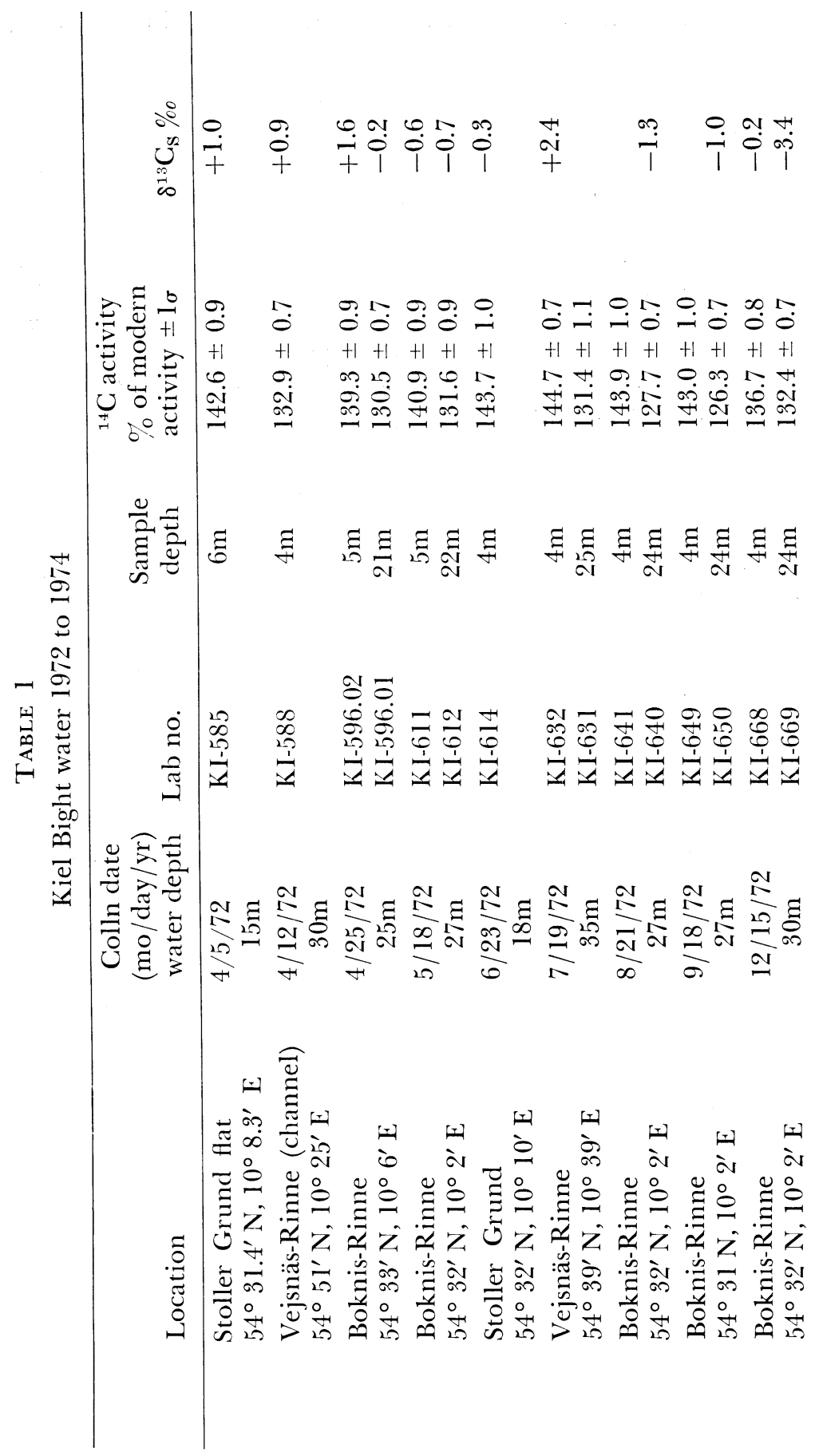


†

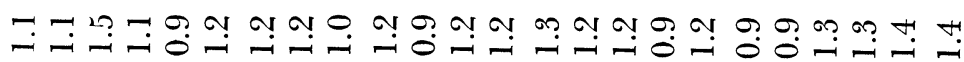
$+1+1+1+1+1+1+1+1+1+1+1+1+1+1+1+1+1+1+1+1+1+1+1+1$ ט.

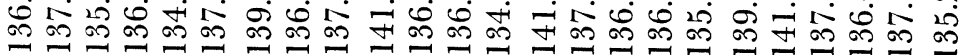

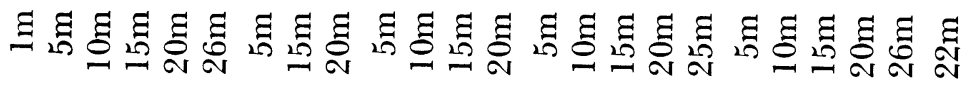



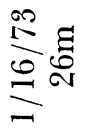

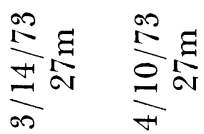

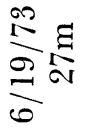



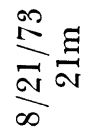

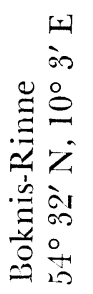

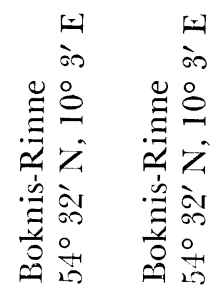

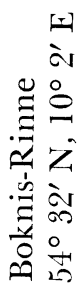

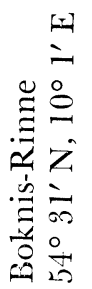




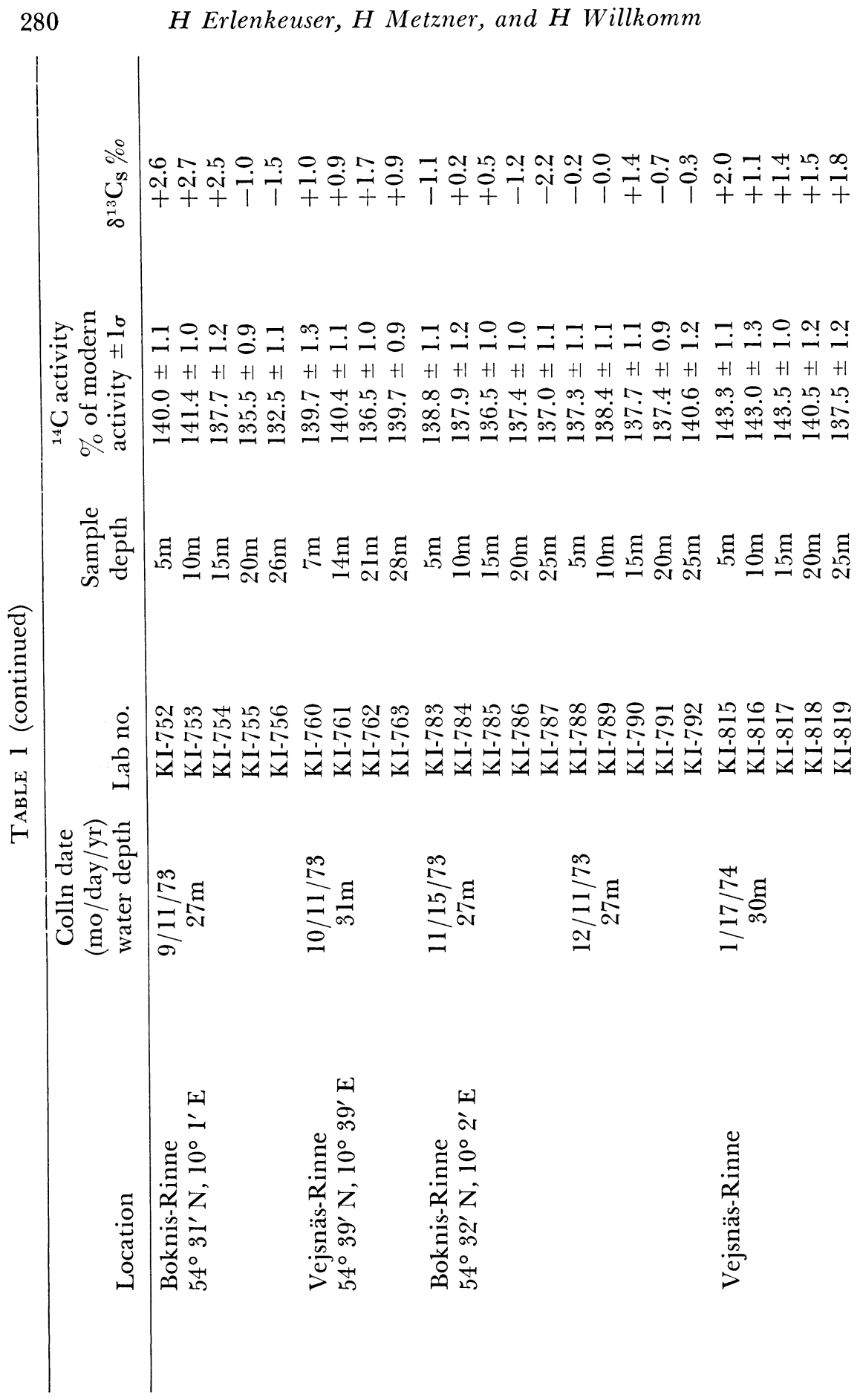




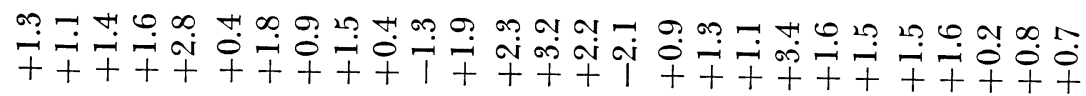


$+1+1+1+1+1+1+1+1+1+1+1+1+1+1+1+1+1+1+1+1+1+1+1+1+1+1+1$

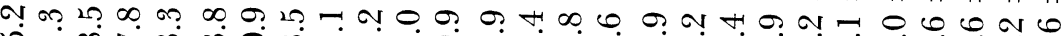

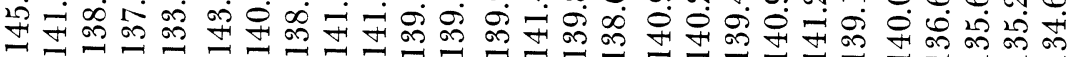

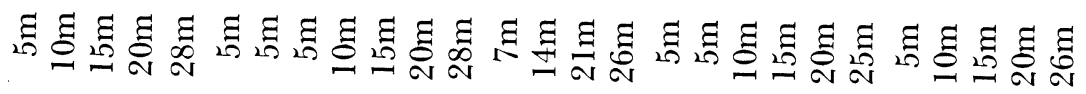

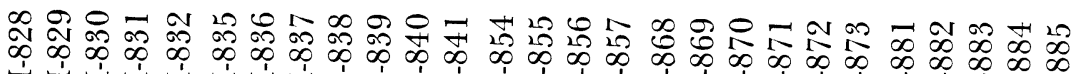

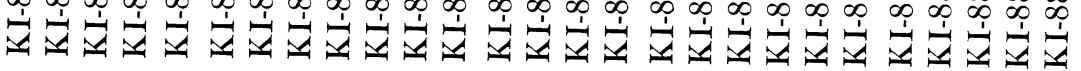

\begin{tabular}{|c|c|c|c|}
\hline 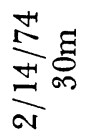 & $\underset{\infty}{\stackrel{N}{\Delta}} \stackrel{\mathbb{N}}{\mathbb{N}}$ & $\begin{array}{l}\stackrel{N}{N}=1 \\
\stackrel{N}{N}\end{array}$ & 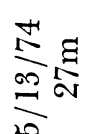 \\
\hline
\end{tabular}

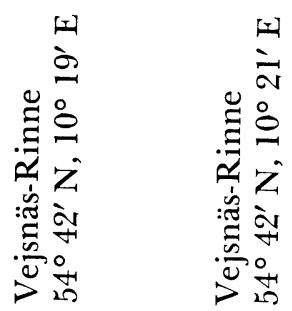

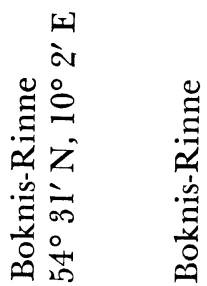

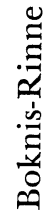


For comparison, some samples from terrestrial biosphere, Baltic Sea, and North Sea were measured.

\section{KI-778. Modern leaves, Summer 1973}

$143.1 \pm 0.7 \%$ $\delta^{13} C_{S}=-26.6 \%$

Leaves (Malus domesticus) coll Oct 1973 near Kiel $\left(54^{\circ} 18^{\prime} 27^{\prime \prime} \mathrm{N}\right.$, $\left.10^{\circ} 4^{\prime} 13^{\prime \prime} \mathrm{E}\right)$.

\section{Baltic Sea series}

Surface water coll in open sea.

\section{KI-696.02. Bornholm 1}

$$
148.0 \pm 0.8 \%
$$

$25 \mathrm{~km} \mathrm{~W}$ of Bornholm I. $\left(55^{\circ} 11.5^{\prime} \mathrm{N}, 14^{\circ} 15.5^{\prime} \mathrm{E}\right)$. Coll and subm March 1973 by H J Black, Inst Meereskunde, Kiel.

\section{KI-862. Bornholm 2}

$\mathbf{1 5 0 . 5} \pm \mathbf{0 . 9 \%}$ $\delta^{13} C_{S}=+2.7 \%$

$20 \mathrm{~km}$ NE of Bornholm I. (55 $\left.19.0^{\prime} \mathrm{N}, 15^{\circ} 14.0^{\prime} \mathrm{E}\right)$. KI-862-865 coll and subm April 1974 by Erwin Suess, Geol-Paläontol Inst, Univ Kiel.

KI-863. Bornholm 3

$146.9 \pm 1.3 \%$

$5 \mathrm{~km}$ SE of Schonen, S Sweden $\left(55^{\circ} 24.5^{\prime} \mathrm{N}, 14^{\circ} 21.3^{\prime} \mathrm{E}\right)$.

\section{KI-864. Bornholm 4}

$149.5 \pm 1.1 \%$

$100 \mathrm{~km}$ NE of Bornholm I. (55 $\left.53.1^{\prime} \mathrm{W}, 16^{\circ} 20.0^{\prime} \mathrm{E}\right)$.

\section{KI-865. Mecklenburg Bight 1}

$30 \mathrm{~km}$ E of Fehmarn I. (54 $\left.24.3^{\prime} \mathrm{N}, 1^{\circ} 50.0^{\prime} \mathrm{E}\right)$.

General Comment: after isotopic correction KI-696.02, -862, and -864 show same activity as modern terrestrial biosphere. This high ${ }^{14} \mathrm{C}$ level probably results from $\mathrm{CO}_{2}$ exchange with atmosphere. Response time of the Baltic as to atmospheric ${ }^{14} \mathrm{C}$ variations seems to be ca $10 \mathrm{yr}$, but too little is known for more comprehensive analysis. Activities are higher than in Kiel Bight. KI-865, Mecklenburg Bight, is clearly affected by Kattegat water, of Kiel Bight series. KI-863 may represent water from Mecklenburg Bight drifting to the $\mathrm{N}$ or may reflect hard water effect of river discharge affecting coastal waters off Sweden.

\section{Bornholm benthos series}

Algae and mussels coll March 1973 by H J Black, 200m off shore of Bornholm I. $\left(55^{\circ} 16.3^{\prime} \mathrm{N}, 14^{\circ} 49.0^{\prime} \mathrm{E}\right)$ at 2 to $4 \mathrm{~m}$ water depth. Comment: correction for isotopic effects gives same activity as water sample, KI696.02.

\section{KI-697. Bornholm, Fucus serratus}

$$
\begin{array}{r}
146.5 \pm 0.7 \% \\
\delta^{13} C_{S}=-16.6 \%
\end{array}
$$


KI-698.01. Bornholm, Furcellaria

Furcellaria fastigiata, plant.

KI-698.02. Bornholm, Furcellaria

Furcellaria fastigiata, roots.

\section{KI-699. Bornholm, Ceramium}

\section{KI-700.001. Bornholm, Mytilus}

Shells (Mytilus edulis). Water depth $5 \mathrm{~m}$.
$144.7 \pm 0.6 \%$ $\delta^{13} C_{S}=-21.4 \%$

$143.3 \pm 0.7 \%$ $\delta^{13} C_{S}=-23.7 \%$

$143.7 \pm 0.8 \%$ $\delta^{13} C_{S}=-21.7 \%$ $152.0 \pm 0.7 \%$ $\delta^{19} C_{S}=-1.6 \%$

\section{Kattegat benthos series}

\section{KI-733. Kattegat, Algae 1}

$126.8 \pm 0.7 \%$

$\delta^{13} C_{S}=-19.5 \%$

Algae coll July 1973 by R R Dries, Inst Meereskunde, Univ Kiel, $18 \mathrm{~km} \mathrm{SE}$ of Skagen $\left(57^{\circ} 38.7^{\prime} \mathrm{N}, 10^{\circ} 51.5^{\prime} \mathrm{E}\right)$. Water depth $19 \mathrm{~m}$, dredge sample.

\section{KI-764. Kattegat, Algae 2}

$126.9 \pm 0.6 \%$

Algae coll Oct 1973 by R R Dries, $25 \mathrm{~km}$ W of Tjörn $\left(57^{\circ} 59^{\prime} \mathrm{N}, 11^{\circ}\right.$ $7.5^{\prime} \mathrm{E}$ ). Water depth $110 \mathrm{~m}$. Different kinds of algae, 2 or more yr old.

\section{KI-765. Kattegat, Algae 3}

$$
\begin{gathered}
126.2 \pm 0.7 \% \\
\delta^{13} C_{S}=-29.4 \%
\end{gathered}
$$

Algae coll Oct 1973 by R R Dries in N entrance of Great Belt channel (55 $\left.53.4^{\prime} \mathrm{N}, 10^{\circ} 54.5^{\prime} \mathrm{E}\right)$. Water depth $14 \mathrm{~m}$. Annual algae, grown Spring 1973.

\section{KI-734.001. Kattegat, Modiolus}

$105.2 \pm 0.5 \%$ $\delta^{13} C_{S}=+1.8 \%$

Shells (Modiolus), 65 to $90 \mathrm{~mm}$ length; coll July 1973 by R R Dries in central Kattegat $\left(56^{\circ} 10.2^{\prime} \mathrm{N}, 11^{\circ} 46.0^{\prime} \mathrm{E}\right)$. Water depth $40 \mathrm{~m}$. Comment: small atomic bomb effect is comparable to that of Cyprina islandica (see below).

\section{B. Benthic samples from Kiel Bight}

\section{Algae, sponges, starfish}

Organic part of specimens coll in SW part of Kiel Bight during 1972 (cf Table 2). Comment: after correction for isotopic effects, activity corresponds to ${ }^{14} \mathrm{C}$ content of water environment (see Table 1).

\section{Kiel Bight mussels (except Cyprina I.)*}

Flesh and shells of living specimens, coll by ${ }^{14} \mathrm{C} \mathrm{Lab}$, in $\mathrm{SW}$ and medium part of Kiel Bight, 1972 to 1974.

* In KI-nos, usually the 3rd decimal describes the kind of sample: 0 or no digit means organic component, I refers to carbonates. Exception: water samples have no subdivision though referring to inorganic $\mathrm{CO}_{2}$. 


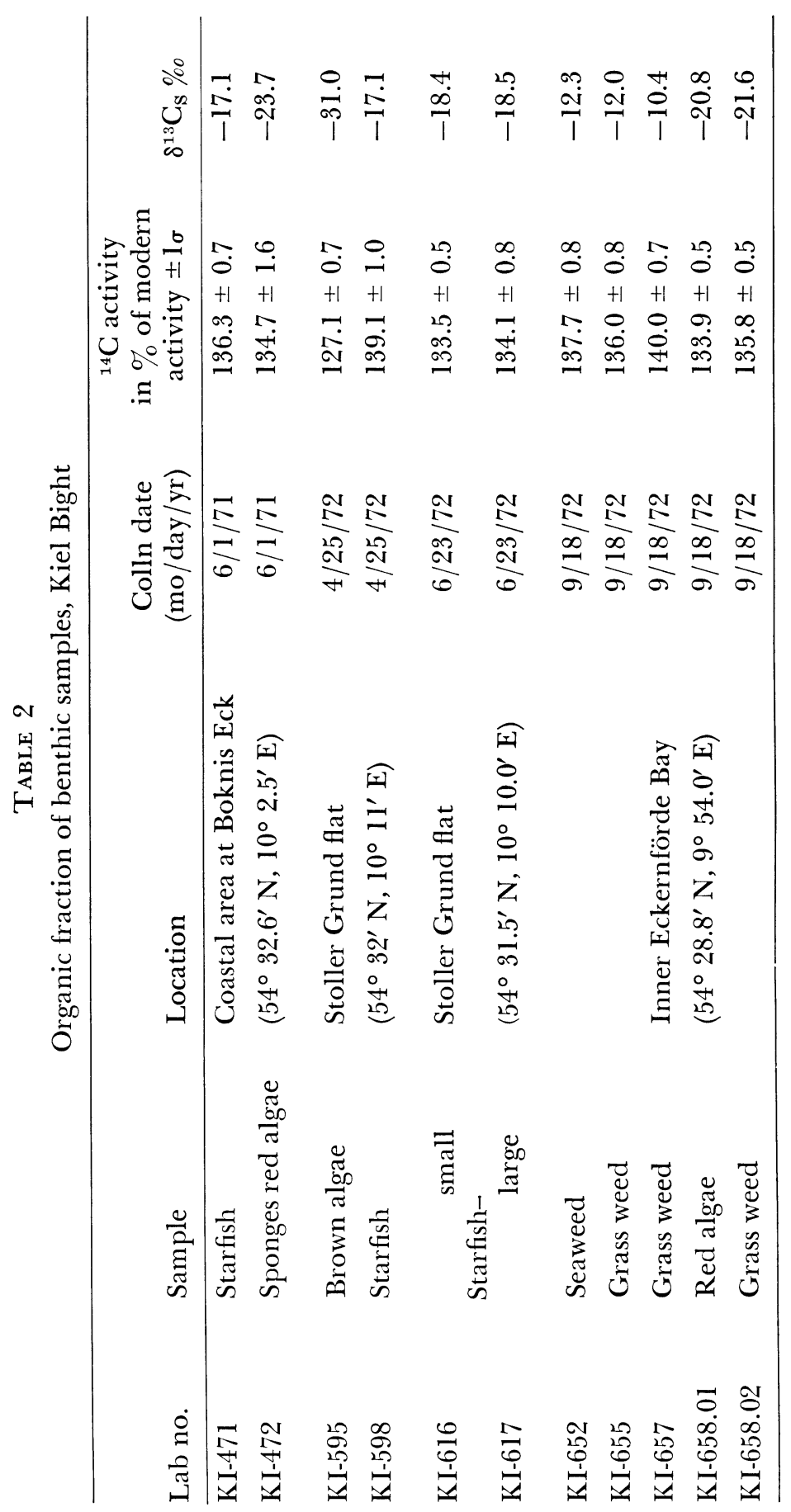




\section{KI-474. Boknis Eck, 710601}

$128.4 \pm 1.1 \%$ $\left.10^{\circ} 2.5^{\prime} \mathrm{E}\right)$ SW Kiel $\left.10^{\circ} 2.5^{\prime} \mathrm{E}\right)$, SW Kiel Bight, $11 \mathrm{~m}$ water depth. Samples were buried by $5 \mathrm{~cm}$ coarse sand. Coll June 1971 by divers of Kiel Univ, subm by $\mathrm{H}$ Kudrass, Geol-Palaeontol Inst, Univ Kiel. Mussels were washed, killed in hot water, shells and flesh were separated and dried; only flesh was studied.

\section{KI-597.001. Stoller Grund, 7204 25-3}

$134.0 \pm 0.7 \%$

Mya arenaria, $11^{\prime}$ E) at -6 to $-8 \mathrm{~m}$ on sand substrat; carbonate fraction $\left(54^{\circ} 32^{\prime} \mathrm{N}, 10^{\circ}\right.$ tivity is lower than in present surface water. This may indicate shell age of ca 5 to $10 \mathrm{yr}$ (see KI-833 f).

\section{KI-599.001. Stoller Grund, 72 04 25-5}

$132.0 \pm 0.7 \%$ $\delta^{13} C_{S}=+0.3 \%$

Shells (Abra alba), coll April $1972 \mathrm{~W}$ of Stoller Grund flat $\left(54^{\circ} 32.1^{\prime}\right.$ $\left.\mathrm{N}, 10^{\circ} 10.3^{\prime} \mathrm{E}\right)$ at $-18 \mathrm{~m}$ with van Veen grab sampler. Comment: life span is known to be only 1 to 2 yr. Activity agrees well with that of water depth in Spring 1972.

\section{KI-613. Boknis Eck, 7205 18-3a}

Astarte borealis from shoreline at Boknis Eck $\left(54^{\circ} 31.5^{\prime} \mathrm{N}, 10^{\circ} 5.0^{\prime} \mathrm{E}\right)$ W Kiel Bight, coll May 1972, at -18 to $-10 \mathrm{~m}$ with van Veen grab. Silt and sand fraction of grab samples were flushed on a $.5 \mathrm{~mm}$ mesh, residue was washed and dried; intact specimens were picked out.

\section{KI-613.011.}

Shells ca $20 \mathrm{~mm}$ diam, carbonate fraction.

\section{KI-613.021.}

Shells, 7 to $17 \mathrm{~mm}$ diam (mean $11 \mathrm{~mm}$ ).

$$
104.8 \pm 0.6 \%
$$$$
\delta^{13} C_{S}=-0.8 \%
$$

$$
136.6 \pm 0.5 \%
$$$$
\delta^{13} C_{S}=+0.8 \%
$$

General Comment: low activity of KI-613.011 is probably due to high mussel life span; considerable part of shell carbonate was secreted before bomb produced ${ }^{14} \mathrm{C}$ entered the water environment since 1963. Activity figure points to mussel age of 10 to $15 \mathrm{yr}$, whereas group of small specimens (KI-613.021) is not older than 5 to 6 yr (cf Cyprina series, below).

\section{KI-615. Mytilus, Stoller Grund, 720623}

Mytilus edulis, W of Stoller Grund flat $\left(54^{\circ} 31.5 \mathrm{~N}, 10^{\circ} 10.0^{\prime} \mathrm{E}\right)$, coll June 1972 at $-18 \mathrm{~m}$; dredge sample. Mussels washed, killed in hot water, flesh and shells separated and dried.

KI-615.011.

$140.5 \pm 0.8 \%$

Shells, 63 to $84 \mathrm{~mm}$ (mean $71.7 \mathrm{~mm}$ ) length, carbonate fraction. 
KI-615.010

Flesh from KI-615.011.

\section{KI-615.021}

Shells, 16 to $30 \mathrm{~mm}$ (mean $22.2 \mathrm{~mm}$ ), carbonate fraction.
$132.4 \pm 0.6 \%$ $\delta^{13} C_{S} c a-22 \%$

$139.1 \pm 0.8 \%$ $\delta^{13} C_{S} \mathrm{caO} \%$

General Comment: carbonate activities agree well with present surface water activity. Mussels are probably shifted from Stoller Grund flat to greater depths by wave action (Samtleben, 1973). Age of larger specimens (KI-615.011) should be ca 5 yr, and water activity seems to have been essentially the same within that time.

\section{Central Kiel Bight mussels}

Benthic faunae and coarse grain fractions from surface layer of muddy sediments $\mathrm{S}$ of entrance of Great Belt channel into Kiel Bight $\left(54^{\circ}\right.$ $\left.36.6^{\prime} \mathrm{N}, 10^{\circ} 37.9^{\prime} \mathrm{E}\right),-18 \mathrm{~m}$. Coll July 1972 with van Veen grab sampler. Same treatment as KI-613.

KI-630.011.

$111.1 \pm 0.7 \%$ $\delta^{13} C_{S}=-0.2 \%$ KI-613.

Astarte borealis, 15 to $25 \mathrm{~mm}$ diam, carbonate fraction. Comment: cf

KI-633.021.

$108.2 \pm 0.5 \%$

Undefined shells and shell fraoments, 1.8 to $2.5 \mathrm{~mm}$ linear dimension.

\section{KI-633.031.}

$124.2 \pm 1.1 \%$

Undefined shells and shell fragments, smaller than $1.8 \mathrm{~mm}$.

General Comment: foraminifera studies yield evidence that carbonates are readily dissolved in muddy sediments of Kiel Bight (Seibold et al, 1971). KI-633 indicates that a major fraction of coarse shell fragments still present in sediment was formed in the pre-bomb era. Part of these carbonates will be probably supplied from the high biomass of long living Cyprina islandica and different Astarte species (Arntz, 1971).

KI-633.051.

Astarte montagui, 2 to $4 \mathrm{~mm}$ diam.

KI-633.061.

Astarte elliptica, up to $25 \mathrm{~mm}$ diam.
$111.5 \pm 0.7 \%$ $\delta^{13} C_{S}=-1.6 \%$

$113.0 \pm 1.1 \%$ $\delta^{13} C_{S}=+0.8 \%$

General Comment: as seen from Cyprina islandica series, low ${ }^{14} \mathrm{C}$ activity must be attributed to long life span of mussels that should markedly exceed $10 \mathrm{yr}$ in this case. 
KI-651.001. Benno 7209 18-1

$140.6 \pm 0.7 \%$

Shells (Mytilus edulis), coll Sept 1972 in interior Eckernförde Bay ( $\left.54^{\circ} 28.8^{\prime} \mathrm{N}, 9^{\circ} 54.0^{\prime} \mathrm{E}\right)$, SW Kiel Bight.

\section{KI-656.001. Benno 7209 18-7}

$140.0 \pm 0.7 \%$

$\delta^{13} C_{S}=+1.0 \%$

Small fragments of mussel shells sieved from surface sediment, coll with KI-651.001.

\section{KI-852.001.}

$140.7 \pm 0.8 \%$

$\delta^{13} C_{S}=-0.7 \%$

Shells (Mytilus edulis) coll Feb 1971 by C Samtleben, Geol Inst Univ Kiel, in Gelting Bay (54 47.0' N, $\left.9^{\circ} 53.7^{\prime} \mathrm{E}\right)$.

\section{Floating substrata series}

Semiramis experiment (Sarnthein \& Richter, 1974). Different substrata (sand, gravel, clay) of ca $\mathrm{lm}^{2}$ surface were exposed in definite heights above ground in the "Hausgarten" ("dooryard") of the SFB 95 near the shore of Boknis Eck ( $54^{\circ} 33^{\prime} \mathrm{N}, 10^{\circ} 3^{\prime} \mathrm{E}$ ). The virginal substrata were populated by larvae and specimens that grew definitely during time of exposure. Activities (Table 3) compare well with average water activities at corresponding depth during that time.

\section{Floating plates, Summer 1973}

Plates of several $\mathrm{dm}^{2}$ surface (KI-776 to 771 , Table 3) were exposed from June to Oct 1973 by $\mathrm{W}$ Richter at same position as Semiramis experiment.

\section{KI-853.001.}

$138.7 \pm 0.7 \%$

$$
\delta^{13} C_{S}=0.0 \%
$$

Shells (Mytilus edulis) coll alive March 1974 by C Samtleben, Geol Palaeontol Inst Univ Kiel on Stoller Grund flat $\left(54^{\circ} 31^{\prime} 53^{\prime \prime} \mathrm{N}, 10^{\circ} 10^{\prime}\right.$ $26^{\prime \prime}$ E) SW Kiel Bight at 9m water depth.

KI-866.001.

$$
\begin{array}{r}
900 \pm 29 \\
\text { AD } 1050 \\
\delta^{13} C_{S}=+0.8 \%
\end{array}
$$

Shells (Cardium edule), $.8 \mathrm{~m}$ below floor, from farm land at coast of Gelting Bay ( $54^{\circ} 46^{\prime} \mathrm{N}, 9^{\circ} 53^{\prime} \mathrm{E}$ ), NW Kiel Bight. Coll and subm by C Samtleben. Shells were found in situ in medium-grained sand layer below clayey fine sand and recent top soil humus layers. Comment: area was diked at AD 1829. Sample indicates higher water level at AD 1000 (Voss, 1970).

\section{Cyprina islandica series}

Shell carbonate and flesh of Cyprina islandica coll alive at different sites in Kiel Bight, W Baltic Sea. Adult specimens had far lower activities than present bottom water bicarbonate (Erlenkeuser \& Willkomm, 1973b). This might be due to long life span of mussel or might indicate partial 
uptake of pre-bomb carbon from sediment, either as carbonate or organic carbon.

\section{KI-586. Gabelsflach $4 / 72$}

Samples, 40 to $60 \mathrm{~mm}$ diam, from muddy sediment $\mathrm{N}$ of Gabelsflach flat $\left(54^{\circ} 37.0^{\prime} \mathrm{N}, 10^{\circ} 21.8^{\prime} \mathrm{E}\right)$ medium Kiel Bight, $21 \mathrm{~m}$ water depth. Coll April 1972 with van Veen grab.

\section{KI-586.000. Flesh}

KI-586.001. Shell carbonate

$$
127.4 \pm 0.5 \%
$$$$
\delta^{13} C_{S}=-20.7 \%
$$

$108.7 \pm 0.7 \%$ $\delta^{13} C_{S}=+0.9 \%$

\section{KI-618. Dorschgrund 6/72}

Cyprinae, 40 to $60 \mathrm{~mm}$, from clayey mud in Dorschgrund basin $\left(54^{\circ}\right.$ $\left.37^{\prime} \mathrm{N}, 10^{\circ} 20^{\prime} \mathrm{E}\right)$ medium Kiel Bight, $21 \mathrm{~m}$ water depth. Coll June 1972 with van Veen grab. $\delta^{13} \mathrm{C}$ not measured.

KI-618.000. Flesh

$131.5 \pm 0.8 \%$

KI-618.001. Shell carbonate

$$
109.2 \pm 0.6 \%
$$

TABLE 3

\begin{tabular}{|c|c|c|c|c|}
\hline Lab no. & Sample & $\begin{array}{l}\text { Depth } \\
\text { of plate }\end{array}$ & $\begin{array}{l}{ }^{14} \mathrm{C} \text { activity, } \\
\% \text { of modern } \\
\text { activity } \pm 1 \sigma\end{array}$ & $\delta^{13} \mathrm{C}_{\mathrm{S}}(\% 0)$ \\
\hline \multicolumn{5}{|c|}{ Semiramis experiment } \\
\hline KI-680.021 & $\begin{array}{l}\text { Mytilus edulis, } \\
\text { 24-31mm long }\end{array}$ & $10 \mathrm{~m}$ & $136.9 \pm 0.9$ & -0.1 \\
\hline KI-680.031 & Balanus & $10 \mathrm{~m}$ & $140.1 \pm 0.9$ & 0.0 \\
\hline KI-680.051 & $\begin{array}{l}\text { Mytilus \& Balanus } \\
\text { small samples }\end{array}$ & $10 \mathrm{~m}$ & $138.8 \pm 0.6$ & +1.9 \\
\hline KI-679.021 & $\begin{array}{l}\text { Mytilus edulis } \\
\text { larger samples } \\
\text { 18-23mm long }\end{array}$ & $15 \mathrm{~m}$ & $137.4 \pm 0.6$ & +0.8 \\
\hline KI-679.031 & Balanus & $15 \mathrm{~m}$ & $137.3 \pm 0.7$ & -0.5 \\
\hline \multicolumn{5}{|c|}{ Floating plates, Summer 1973} \\
\hline KI-766.001 & Mytilus edulis & $4 \mathrm{~m}$ & $139.4 \pm 0.7$ & +0.8 \\
\hline KI-767.001 & " & $7 \mathrm{~m}$ & $139.7 \pm 0.6$ & -0.1 \\
\hline KI-768.001 & $"$ & $10 \mathrm{~m}$ & $138.6 \pm 0.6$ & +1.6 \\
\hline KI-769.001 & $"$ & $14 \mathrm{~m}$ & $139.1 \pm 0.7$ & -0.1 \\
\hline KI-770.001 & Balanus & $16 \mathrm{~m}$ & $139.1 \pm 0.7$ & -0.1 \\
\hline KI-771.001 & & $18 \mathrm{~m}$ & $137.9 \pm 0.6$ & +1.1 \\
\hline
\end{tabular}

Floating substrata series 
KI-629. Millionenviertel 7/72

Samples 50 to $70 \mathrm{~mm}$ diam from muddy sand substrate of Millionenviertel $\left(54^{\circ} 36.6^{\prime} \mathrm{N}, 10^{\circ} 37.9^{\prime} \mathrm{E}\right)$. Coll July 1972 with van Veen grab.

\section{KI-629.011. Shell carbonate}

$$
\begin{gathered}
\mathbf{1 1 0 . 4} \pm \mathbf{0 . 7} \% \\
\delta^{13} C_{S}=+1.4 \% \\
\mathbf{1 2 0 . 5} \pm \mathbf{0 . 6} \% \\
\delta^{13} C_{S}=-20.0 \%
\end{gathered}
$$

KI-629.020. Flesh

\section{KI-629.021. Shell carbonate}

$$
128.8 \pm 0.7 \%
$$

$$
\delta^{18} C_{S}=+0.5 \%
$$

Outer rim, ca $10 \mathrm{~mm}$ wide, of different shells. Comment: note higher ${ }^{14} \mathrm{C}$ activity in more recent parts of shells (cf KI-833, -843).

\section{KI-633.081. Millionenviertel, $7 / 72$}

$133.1 \pm 0.7 \%$ $\delta^{13} C_{S}=+0.7 \%$

Shell carbonate of smaller samples, 0.5 to $3.4 \mathrm{~mm}$ from muddy sand of Millionenviertel area, $S$ of Great Belt channel entrance into Kiel Bight $\left(54^{\circ} 36.6^{\prime} \mathrm{N}, 10^{\circ} 37.9^{\prime} \mathrm{E}\right), 18 \mathrm{~m}$ water depth. Coll July 1972 with van Veen grab. Comment: activity is markedly higher than for large Cyprina specimens (KI-586, -618, -629).

\section{KI-833. Dorschgrund 2/74}

Cyprinae dredged along transect in Dorschgrund area, a shallow basin with muddy sediments in medium Kiel Bight $\left(54^{\circ} 36.5^{\prime} \mathrm{N}, 10^{\circ} 19.3^{\prime}\right.$ $\mathrm{E}$ to $\left.54^{\circ} 37.4^{\prime} \mathrm{N}, 10^{\circ} 19.6^{\prime} \mathrm{E}\right), 21 \mathrm{~m}$ water depth. Coll Feb 1974. Shells of 50 to $70 \mathrm{~mm}$ diam were picked out and each cut into sector segments parallel to growth lines proceeding from outer rim towards hinge. Ring width is $5 \mathrm{~mm}$, measured on a sec at greatest distance between hinge and rim. Corresponding segments of the different shells were combined; carbonate fraction studied.

KI-833.011. 1st segment 0 to $5 \mathrm{~mm}$ from rim.

\section{KI-833.021. 2nd segment} 6 to $10 \mathrm{~mm}$ from rim.

\section{KI-833.031. 3rd segment} 11 to $15 \mathrm{~mm}$ from rim.

KI-833.041. 4th segment 16 to $20 \mathrm{~mm}$ from rim.

KI-833.051. 5th segment 21 to $25 \mathrm{~mm}$ from rim.
$133.8 \pm 0.6 \%$ $\delta^{13} C_{S}=-0.7 \%$

$$
111.9 \pm 0.6 \%
$$
$\delta^{13} C_{S}=-0.7 \%$

$102.6 \pm 0.6 \%$ $\delta^{13} C_{S}=-4.2 \%$ $\delta^{13} C_{S}=+0.8 \%$

$100.3 \pm 0.8 \%$ $\delta^{13} C_{S}=+1.1 \%$ 。 
KI-833.061. 6th segment

26 to $30 \mathrm{~mm}$ from rim.

KI-833.071. Remnant hinge parts

Smaller $25 \mathrm{~mm}$.

KI-833.081. Remnant hinge parts

26 to $36 \mathrm{~mm}$.
$105.1 \pm 0.8 \%$ $\delta^{13} C_{S}=+0.7 \%$

$104.9 \pm 0.7 \%$ $\delta^{13} C_{S}=-0.1 \%$

$105.1 \pm 0.6 \%$ $\delta^{13} C_{S}=+0.7 \%$

\section{KI-843.}

Cyprina islandica, same position and treatment as KI-833. Coll March 1974. 82 shells, diam within 49 to $51 \mathrm{~mm}$, selected and cut as before.

KI-843.011. Ist segment

0 to $5 \mathrm{~mm}$ from rim.

KI-843.021. 2nd segment

6 to $10 \mathrm{~mm}$ from rim.

KI-843.031. 3rd segment

11 to $15 \mathrm{~mm}$ from rim.

KI-843.041. 4th segment

16 to $20 \mathrm{~mm}$ from rim.

KI-843.051. 5th segment

21 to $25 \mathrm{~mm}$ from rim.

KI-843.061. 6th segment

26 to $30 \mathrm{~mm}$ from rim.

KI-843.071.

Hinge plates, without hinge bands, 30 to $45 \mathrm{~mm}$ from rim.

KI-843.081. Hinge bands

\section{KI-843.09}

Smaller samples of KI-843, 11 to $30 \mathrm{~mm}$ diam (mean diam weighted by shell weight: $25.5 \mathrm{~mm}$ ).

KI-843.090. Flesh

KI-843.091. Shell carbonate
$137.2 \pm 0.7 \%$ $\delta^{13} C_{S}=+0.7 \%$

$116.6 \pm 0.7 \%$ $\delta^{13} C_{S}=+1.1 \%$

$102.5 \pm 0.6 \%$ $\delta^{13} C_{s}=+0.5 \%$

$103.0 \pm 0.5 \%$ $\delta^{13} C_{S}=+0.5 \%$

$102.9 \pm 0.6 \%$ $\delta^{13} C_{S}=+1.6 \%$ 。

$104.9 \pm 0.6 \%$ $\delta^{13} C_{S}=+1.0 \%$

$106.7 \pm 0.7 \%$ $\delta^{13} C_{S}=+1.5 \%$

$108.8 \pm 0.6 \%$ $\delta^{13} C_{S}=+0.5 \%$ 。 
KI-851.001. Vejsnäs flat $12 / 71$

$135.7 \pm 0.7 \%$

Cyprina islandica from Vejsnäs flat $\left(54^{\circ} 47^{\prime} \mathrm{N}, 10^{\circ} 28^{\prime} \mathrm{E}\right) \mathrm{N}$ Kiel Bight. Coll Dec 1971 by C Samtleben and subm April 1974 by Samtleben and Lohr, ${ }^{14} \mathrm{C}$ Lab, Kiel. Mussels were coll alive with van Veen grab. Samples smaller than $31 \mathrm{~mm}$ were selected for analysis of shell carbonate. General Comment: ${ }^{14} \mathrm{C}$ variations of shell carbonate reflect increase of ${ }^{14} \mathrm{C}$ level in marine environment since ca 1960 . Outer parts of ca $15 \mathrm{~mm}$ width (KI-629, -833, -843) as well as small samples (KI-843.09, -851, -633.08) grew during last decade. Growth rate of samples up to 30 to $40 \mathrm{~mm}$ diam is ca $3 \mathrm{~mm} / \mathrm{yr}$ (Kühlmorgen-Hille, 1962) so that total mussel age is ca $20 \mathrm{yr}$. Increasing ${ }^{14} \mathrm{C}$ activity towards hinge indicates gradual thickening during recent growth period. Medium parts of shells estimate pre-bomb ${ }^{14} \mathrm{C}$ level in Kiel Bight bottom water. A more detailed analysis is being conducted based on biometrical studies. High activity of KI843.090 indicates most food originates from plankton from surface water. Older organic constituents with ${ }^{14} \mathrm{C}$ level lower than $100 \%$ found in uppermost layer of sediment (Erlenkeuser et al, 1974) do not seem nutritions. Flesh activity of adult samples (KI-586, -618, -629) is generally higher than carbonate activity, due to incorporation of high ${ }^{14} \mathrm{C}$ food by mussel.

\section{Sediment pretreatment studies}

Kiel Bight sediments contain organic constituents of different age (Erlenkeuser et al, 1974) at possibly different stage of diagenesis. Preliminary studies were performed to check effect of chemical pretreatment on ${ }^{14} \mathrm{C}$ age.

KI-600. Stoller Grund, 720425-6

93.4 $\pm 0.5 \%$

$\delta^{13} C_{S}=-22.4 \%$

Organic component of muddy surface sediment coll April 1972 at Stoller Grund ( $\left.54^{\circ} 32.0^{\prime} \mathrm{N}, 10^{\circ} 10.0^{\prime} \mathrm{E}\right)$. Water depth $18 \mathrm{~m}$.

KI-604.001. Stoller Grund, 720425-7

$139.6 \pm 1.0 \%$ $\delta^{13} C_{S}=-1.2 \%$

Surface sediment like KI-600. Small-sized fraction was flushed and grain size class greater . $1 \mathrm{~mm}$ was coll; carbonate studied.

\section{KI-610. Aeroe SW, GPI 11777-1}

Muddy sediment from NW Kiel Bight ( $\left.54^{\circ} 46.2^{\prime} \mathrm{N}, 10^{\circ} 11.6^{\prime} \mathrm{E}\right)$, W Baltic Sea. Coll and subm March 1971 by F Werner, Geol-Palaeontol Inst Univ Kiel, H Erlenkeuser, and H Willkomm. Box core from surface sediment, at $-26 \mathrm{~m}$, from same location as KI-483 (Erlenkeuser \& Willkomm, 1973). Upper half (ca $20 \mathrm{~cm}$ ) of core was thoroughly mixed to provide sufficient material for applying different pretreatment procedures and divided in several samples. Samples were prepared differently to study influence of $\mathrm{HCl}$ boiling on organic component. Results below account for water content of sample material. KI-483 (cf Erlenkeuser \& Willkomm, 
1973 ), a core coll $.2 \mathrm{~km} \mathrm{~W}$, indicates that activity of organic component comprises range of 82 to $94 \%$ of standard activity.

\section{KI-610.01. 1hr, 1\% HCl}

$$
\begin{array}{r}
\mathbf{8 7 . 5} \pm \mathbf{0 . 7 \%} \\
\delta^{13} C_{S}=-23.2 \%
\end{array}
$$

$1000 \mathrm{~g}$ wet sediment (ca $300 \mathrm{~g}$ by dry weight), boiled for $1 \mathrm{hr}$ in $1000 \mathrm{ml}$ $1 \% \mathrm{HCl}$ (sample water included), centrifuged and decanted; stirred twice with $800 \mathrm{ml}$ water, centrifuged, decanted, stirred again with water and dialyzed for $6 \mathrm{hr}$ until $\mathrm{pH}=6$. Afterwards, residual traces of $\mathrm{Cl}^{-}$are negligible.

\section{KI-610.02. 2hr, 1\% HCI}

$86.1 \pm 0.8 \%$

$1000 \mathrm{~g}$ wet sediment, boiled for $2 \mathrm{hr}$ in $1000 \mathrm{ml} 1 \% \mathrm{HCl}$, centrifuged, decanted, stirred up with $450 \mathrm{ml}$ water; no washing, dialysis for 6 days.

\section{KI-610.03. 4.5hr, 1\% HCl}

$88.5 \pm 0.9 \%$

$1000 \mathrm{~g}$ wet sediment, boiled for $4.5 \mathrm{hr}$ in $1 \% \mathrm{HCl}, \mathrm{H}_{\mathrm{s}}$ canted, washed by repeated centrifuging and decanting (5 times with a total of $1.6 \mathrm{~L}$ water). Washing procedure did not remove $\mathrm{Cl}^{-}$sufficiently; therefore, this time consuming procedure was replaced by dialysis; sample dialyzed for 6 days.

\section{KI-610.08. 1hr, 0.9\% HCl}

$86.5 \pm 0.6 \%$

$1000 \mathrm{~g}$ wet sediment, boiled for $1 \mathrm{hr}$ in $.9 \% \mathrm{HCl}$, not centrifuged but directly dialyzed for 6 days.

General Comment: $\mathrm{CO}_{2}$ yield by oxidation seems to be correlated to intensity of washing: it was 2.5, 1.9, 1.1, and 1.0 (arbitrary units) for KI$610.08,-610.02,-610.01$ and -610.3 , respectively. However, no statistically significant trend is seen from the ${ }^{14} \mathrm{C}$ data, indicating that sample pretreatment does not affect ${ }^{14} \mathrm{C}$ dates appreciably for this type of sediment.

\section{KI-620.472.}

Sample of core, KI-620 (see Table 7), $96 \mathrm{~cm}$ below sediment surface. Chemical treatment: initial wet weight $1000 \mathrm{~g}$; carbonates removed by hot $2 \% \mathrm{HCl}$; sample centrifuged and decanted; residue leached 3 times with $\ln \mathrm{NaOH}$ at $100^{\circ} \mathrm{C}$ for $5 \mathrm{hr}$, centrifuged, and decanted. Eluate is acidified to precipitate humic acids, which are washed to neutrality on analytic filter and dried. Humic acid yield was $6.8 \mathrm{~g}, 2.6 \mathrm{~g}, 1.1 \mathrm{~g}$ by dry weight for 1st, 2nd, and 3rd leaching procedures, respectively; fractions 1 and 2 were combined for dating. Comment: radiocarbon date fits well sedimentation line obtained from KI-620, indicating, that humic acid fraction is representative for ${ }^{14} \mathrm{C}$ activity of total organic fraction of sediment. 


\section{Sediment cores from Kiel Bight}

\section{Breitgrund channel series}

\section{KI-619. Sediment Core GPI 11881}

Core, $58 \mathrm{~cm}$ long, from bottom of Breitgrund channel, NW Kiel Bight $\left(54^{\circ} 47.4^{\prime} \mathrm{N}, 10^{\circ} 1.7^{\prime} \mathrm{E}\right)$. Coll May 1972 in $32.5 \mathrm{~m}$ water depth with box corer by Friedrich Werner, Geol Palaeontol Inst Univ Kiel. Upper layer, $22 \mathrm{~cm}$ thick was of marine, lower part of limnic origin (with lake marl and plant residues). Core was cut into slices of 1 or $2 \mathrm{~cm}$ (samples .02 to .08 with an inclination of $23^{\circ}$ according to stratigraphic records within sediment), and both organic and carbonate fraction were dated (Table 4). Comment: an 8000-yr hiatus is found between marine and limnic sediments, which must be explained by lack of sedimentation or later erosion rather than by hard water effect of lake lime. Limnic sediment accumulates at a rate of ca $.6 \mathrm{~mm} / \mathrm{yr}$.

\section{KI-621. Sediment Core GPI 11882}

Core from SW slope of Breitgrund channel (54 47.0 $4^{\prime} \mathrm{N}, 10^{\circ} 0.9^{\prime} \mathrm{E}$ ) $1200 \mathrm{~m}$ WSW of KI-619. Coll by F Werner May 1972 at $29 \mathrm{~m}$ water depth with box corer; total length $2.90 \mathrm{~m}$. Below $145 \mathrm{~cm}$ were limnic sediments

TABLE 4

Kiel Bight sediments, Breitgrund channel, GPI $11881 \equiv$ KI-619

\begin{tabular}{|c|c|c|c|c|c|}
\hline \multirow[b]{2}{*}{$\begin{array}{l}\text { Lab } \\
\text { no. }\end{array}$} & \multirow{2}{*}{$\begin{array}{c}\text { Depth } \\
\text { within } \\
\text { sediment } \\
(\mathrm{mm})\end{array}$} & \multicolumn{2}{|c|}{ Organic fraction } & \multicolumn{2}{|c|}{ Carbonate fraction } \\
\hline & & $\begin{array}{l}\text { Libby age } \\
\text { BP } \pm 1 \sigma\end{array}$ & $\delta^{13} \mathrm{C}_{\mathrm{S}}(\% 0)$ & $\begin{array}{l}\text { Libby age } \\
\text { BP } \pm 1 \sigma\end{array}$ & $\delta^{13} \mathrm{C}_{\mathrm{S}}(\% o$ \\
\hline 619.01 & 0 to 40 & $750 \pm 75$ & -19.9 & & \\
\hline 619.02 & 40 to 49 & $940 \pm 70$ & - & & \\
\hline 619.03 & 49 to 58 & $1000 \pm 75$ & -22.1 & & \\
\hline 619.06 & 77 to 87 & $1360 \pm 65$ & -21.6 & & \\
\hline 619.07 & 87 to 106 & $1990 \pm 210$ & -22.6 & & \\
\hline 619.08 & 106 to 125 & $1120 \pm 80$ & -18.4 & & \\
\hline 619.11 & 125 to 155 & $1370 \pm 40$ & -21.7 & & \\
\hline 619.14 & 195 to 215 & $1330 \pm 50$ & -22.2 & & \\
\hline 619.16 & 225 to 245 & $7060 \pm 90$ & -25.9 & $10,420 \pm 190$ & -3.9 \\
\hline 619.18 & 255 to 270 & $10,120 \pm 120$ & -31.1 & $10,060 \pm 180$ & -2.5 \\
\hline 619.22 & 330 to 350 & $10,220 \pm 190$ & -27.6 & $10,520 \pm 130$ & -4.0 \\
\hline 619.26 & 410 to 430 & $10,000 \pm 130$ & -27.8 & $10,330 \pm 100$ & -4.7 \\
\hline 619.28 & 450 to 470 & & & $10,430 \pm 120$ & -1.7 \\
\hline 619.29 & 470 to 490 & & & $9890 \pm 130$ & -1.0 \\
\hline 619.30 & 490 to 510 & $10,560 \pm 210$ & -28.0 & $7930 \pm 70$ & -4.5 \\
\hline 619.31 & 510 to 530 & & & $10,560 \pm 130$ & -1.4 \\
\hline 619.32 & 530 to 550 & $10,740 \pm 200$ & -28.7 & $10,280 \pm 130$ & -0.8 \\
\hline 619.34 & 570 to 586 & $10,920 \pm 240$ & -30.6 & $10,810 \pm 200$ & - \\
\hline
\end{tabular}


with clayey gyttja and peaty layers. Only these sediments were dated (Table 5). From organic component, a mean sedimentation rate of $.3 \mathrm{~mm} / \mathrm{yr}$ is calculated.

\section{KI-659. Sediment Core GPI 11888}

Core from NE slope of Breitgrund channel $\left(54^{\circ} 47.4^{\prime} \mathrm{N}, 10^{\circ} 1.7^{\prime} \mathrm{E}\right.$ ) $30 \mathrm{~m}$ NE of KI-619. Coll by F Werner June 1972 at $30 \mathrm{~m}$ water depth with short box corer. Organic fraction was measured (Table 6).

\section{KI-660. Sediment Core GPI 11889}

Core from bottom of Breitgrund channel (54 $\left.47.4^{\prime} \mathrm{N}, 10^{\circ} 1.5^{\prime} \mathrm{E}\right)$ $170 \mathrm{~m}$ WSW of KI-619. Coll by F Werner June 1972 at $32.5 \mathrm{~m}$ water depth with short box corer. Organic fraction of upper layers was dated (Table 6). General Comment: recent studies on closely spaced samples from another core from bottom of Breitgrund channel indicate that ${ }^{14} \mathrm{C}$ variations in near surface sediments reflect anthropogenic affects as already recognized in Cores KI-620 and -483. Recent sediments then accumulate at only slightly reduced rate of ca $1 \mathrm{~mm} / \mathrm{yr}$ as compared to 2 to $3 \mathrm{~mm} / \mathrm{yr}$ in Kiel Bight basins, but total thickness of Holocene marine sequence is kept down by single erosional events.

\section{KI-620. Sediment Core GPI 11883}

Core from Boknis channel, outer Eckernförde Fjord, Kiel Bight SW $\left(54^{\circ} 31.5^{\prime} \mathrm{N}, 10^{\circ} 1.8^{\prime} \mathrm{E}\right)$. Coll by $\mathrm{F}$ Werner, May 1972 at $29 \mathrm{~m}$ water depth with box corer. Whole core $(199 \mathrm{~cm})$ is of marine origin. Only organic fraction was dated (Table 7). Comment: ${ }^{14} \mathrm{C}$ dates of deeper layers give sedimentation rate of $1.4 \mathrm{~mm} / \mathrm{yr}$ and an (extrapolated) surface age of $850 \pm 50 \mathrm{yr}(90 \pm .5 \%$ of standard activity). However, above $20 \mathrm{~cm}$ depth (depth corresponds to AD 1830 if sedimentation rate is constant up to

TABLE 5

Kiel Bight sediments, Breitgrund channel, GPI $11882 \equiv$ KI-621

\begin{tabular}{|c|c|c|c|c|c|}
\hline \multirow[b]{2}{*}{$\begin{array}{l}\text { Lab } \\
\text { no. }\end{array}$} & \multirow{2}{*}{$\begin{array}{c}\text { Depth } \\
\text { within } \\
\text { sediment } \\
(\mathrm{cm})\end{array}$} & \multicolumn{2}{|c|}{ Organic fraction } & \multicolumn{2}{|c|}{ Carbonate fraction } \\
\hline & & $\begin{array}{c}\text { Libby age } \\
\text { BP } \pm \mathbf{l}_{\sigma}\end{array}$ & $\delta^{13} \mathrm{C}_{\mathrm{S}}(\% o)$ & $\begin{array}{l}\text { Libby age } \\
\text { вP } \pm 1 \sigma\end{array}$ & $\delta^{13} \mathrm{C}_{\mathrm{S}}(\% o)$ \\
\hline 621.091 & 172 to 174 & & & $12,040 \pm 400$ & +1.9 \\
\hline 621.094 & 178 to 180 & $8110 \pm 100$ & -29.6 & $10,570 \pm 140$ & +0.1 \\
\hline 621.099 & 188 to 190 & $8450 \pm 110$ & -28.7 & $9530 \pm 110$ & +2.2 \\
\hline 621.104 & 198 to 200 & $8980 \pm 110$ & -28.5 & $8620 \pm 120$ & +2.0 \\
\hline 621.109 & 208 to 210 & $8990 \pm 75$ & -27.4 & $8370 \pm 110$ & +0.4 \\
\hline 621.114 & 218 to 220 & $9100 \pm 120$ & -28.8 & $8490 \pm 110$ & 0.0 \\
\hline 621.118 & 226 to 228 & & & $8760 \pm 110$ & +1.2 \\
\hline 621.121 & 232 to 234 & & & $9730 \pm 100$ & +1.0 \\
\hline 621.122 & 234 to 236 & $9985 \pm 120$ & -28.0 & $9900 \pm 120$ & -1.0 \\
\hline
\end{tabular}


TABLE 6

Kiel Bight sediments, Breitgrund channel

\begin{tabular}{cccc}
\hline \multicolumn{5}{c}{$\begin{array}{c}\text { Depth within } \\
\text { Lab no. }\end{array}$} & $\begin{array}{c}\text { Libby age } \\
\text { BP } \pm 1 \sigma\end{array}$ & $\delta^{13} \mathbf{C}_{\mathbf{S}}(\%)$ \\
\hline KI-659. & Organic fraction. Core GPI 11888 \\
659.01 & 0 to 1 & $1100 \pm 40$ & -23.6 \\
659.02 & 1 to 2 & $710 \pm 55$ & -23.5 \\
659.03 & 12 to 13 & $1430 \pm 75$ & -23.1 \\
659.04 & 13 to 14 & $1390 \pm 70$ & -22.9 \\
659.08 & 21 to 22 & $2900 \pm 110$ & -24.5 \\
KI-660. & Organic fraction. Core GPI 11889 & \\
660.01 & 0 to 1 & $780 \pm 110$ & -23.9 \\
660.02 & 1 to 2 & $810 \pm 60$ & -23.3 \\
660.04 & 3 to 4 & $1220 \pm 55$ & -21.9 \\
660.06 & 5 to 6 & $1210 \pm 65$ & -22.7 \\
\hline
\end{tabular}

Table 7

Kiel Bight sediments, Boknis channel, organic fraction, Core GPI $11883=\mathrm{KI}-620$

\begin{tabular}{lrrr}
\hline Lab no. & $\begin{array}{c}\text { Depth within } \\
\text { sediment }(\mathrm{cm})\end{array}$ & $\begin{array}{c}\text { Libby age } \\
\mathbf{B P} \pm 1 \boldsymbol{\sigma}\end{array}$ & $\delta^{13} \mathbf{C}_{\mathrm{S}}(\% \circ)$ \\
\hline 620.01 & 0 to 5 & $1930 \pm 90$ & -22.6 \\
620.02 & 5 to 7 & $2120 \pm 70$ & -22.6 \\
620.03 & 7 to 9 & $1450 \pm 70$ & -21.5 \\
620.04 & 9 to 11 & $1290 \pm 55$ & -20.2 \\
620.05 & 11 to 13 & $1400 \pm 75$ & - \\
620.06 & 13 to 15 & $1080 \pm 90$ & -22.5 \\
620.07 & 15 to 17 & $1110 \pm 40$ & -23.6 \\
620.09 & 19 to 21 & $940 \pm 55$ & -21.4 \\
620.11 & 23 to 25 & $930 \pm 40$ & -21.0 \\
620.20 & 41 to 43 & $1160 \pm 60$ & -22.7 \\
620.24 & 49 to 51 & $1210 \pm 50$ & -21.3 \\
620.96 & 73 to 75 & $1490 \pm 60$ & -21.5 \\
620.37 & 75 to 77 & $1290 \pm 40$ & -21.3 \\
620.45 & 91 to 93 & $1500 \pm 40$ & -20.8 \\
620.47 & 95 to 97 & $1450 \pm 55$ & -22.7 \\
620.50 & 101 to 103 & $1560 \pm 40$ & -22.3 \\
620.61 & 123 to 125 & $1670 \pm 70$ & -22.2 \\
620.74 & 149 to 151 & $1860 \pm 65$ & -22.0 \\
620.86 & 173 to 175 & $2490 \pm 60$ & -21.7 \\
620.98 & 197 to 199 & $2820 \pm 75$ & -22.3 \\
\hline
\end{tabular}


surface), ${ }^{14} \mathrm{C}$ activity decreases to $77 \%$ approaching surface, reflecting increasing effect of industrialization ( $c f$ Erlenkeuser et al, 1974). Sediments SW Aeroe ( $c f$ KI-483 in Erlenkeuser \& Willkomm, 1973) showed a similar stratification with respect to ${ }^{14} \mathrm{C}$ activity.

\section{E. Marine sediment cores from different locations}

\section{Great Belt series}

Sediment cores, coll by Friedrich Werner and Kyaw Winn, Nov 1972, both Geol Palaeontol Inst Univ Kiel, with box corer, from Great Belt channel, main inlet to Baltic Sea. Measurements are part of a study on sedimentology and morphology of Great Belt (cf Winn, 1974). ${ }^{14} \mathrm{C}$ dates of organic component of both cores are given in Table 8 .

\section{KI-736. Sediment Core GPI 12522-2}

Core, $132 \mathrm{~cm}$ long, coll in Great Belt $\left(55^{\circ} 22^{\prime} 35^{\prime \prime} \mathrm{N}, 10^{\circ} 56^{\prime} 46^{\prime \prime} \mathrm{E}\right)$ in $27 \mathrm{~m}$ water depth. 0 to $66 \mathrm{~cm}$ marine sediments, 66 to $115 \mathrm{~cm}$ limnic sediments, 115 to $132 \mathrm{~cm}$ peaty gyttja.

\section{KI-738. Sediment Core GPI 12519-2}

Core $286.5 \mathrm{~cm}$ long, coll in $25.5 \mathrm{~m}$ depth $4 \mathrm{~km} \mathrm{NNE}$ of KI-736 $\left(55^{\circ} 24^{\prime}\right.$ $40^{\prime \prime} \mathrm{N}, 10^{\circ} 58^{\prime} 26^{\prime \prime} \mathrm{E}$ ), marine sediments.

\section{KI-847. Sediment Core GPI 12549-1}

$$
\begin{gathered}
\mathbf{1 0 6 0} \pm \mathbf{7 5} \\
\mathbf{A D ~ 8 9 0} \\
\delta^{13} C_{S}=-19.8 \%
\end{gathered}
$$

Coll Jan 1973 by Heinz Lange and Kyaw Winn, both Geol Palaeont Inst Univ Kiel, in Great Belt channel $17 \mathrm{~km} \mathrm{~N}$ of KI-736 $\left(55^{\circ} 32.5^{\prime} \mathrm{N}\right.$, $10^{\circ} 57.9^{\prime} \mathrm{E}$ ) in $27 \mathrm{~m}$ water depth. Organic component between 25 and $35 \mathrm{~cm}$ depth within sediment was dated (cf Winn, 1974).

\section{KI-848. Sediment Core GPI 10511}

Core, $236 \mathrm{~cm}$ long, coll by Kyaw Winn at $\mathrm{S}$ exit of Great Belt channel, $8 \mathrm{~km} \mathrm{~S}$ of Langeland I. (54 $39.7^{\prime} \mathrm{N}, 10^{\circ} 45.6^{\prime} \mathrm{E}$ ) in $26 \mathrm{~m}$ depth with vibrocorer. Results are given in Table 8.0 to $210 \mathrm{~cm}$ sediment are sand, 210 to $236 \mathrm{~cm}$ are gyttja. Comment: mean sedimentation rate is $.30 \mathrm{~mm} / \mathrm{yr}$.

\section{KI-563. Landsort Basin Core GPI 10076-3}

Sediments from Landsort Basin (58 40.1 $1^{\prime} \mathrm{N}, 18^{\circ} 19.2^{\prime} \mathrm{E}$ ), middle Baltic Sea. Coll Jan 1971 and subm by Erwin Suess, Geol Palaeont Inst Univ Kiel. Measurements of organic and carbonate component (Table 9) continue studies on same core publ under KI-405 (Erlenkeuser \& Willkomm, 1973). Comment: KI-563.02 and -563.03 do not fit preceding results of KI-405. $\delta^{13} \mathrm{C}$ values of carbonate are unusually low compared to carbon isotope ratios of recent Baltic surface water. Same ${ }^{14} \mathrm{C}$ activity of organic and carbonate indicates diagenetic effects to be important for carbonate formation. 
TABLE 8

Great Belt channel sediments

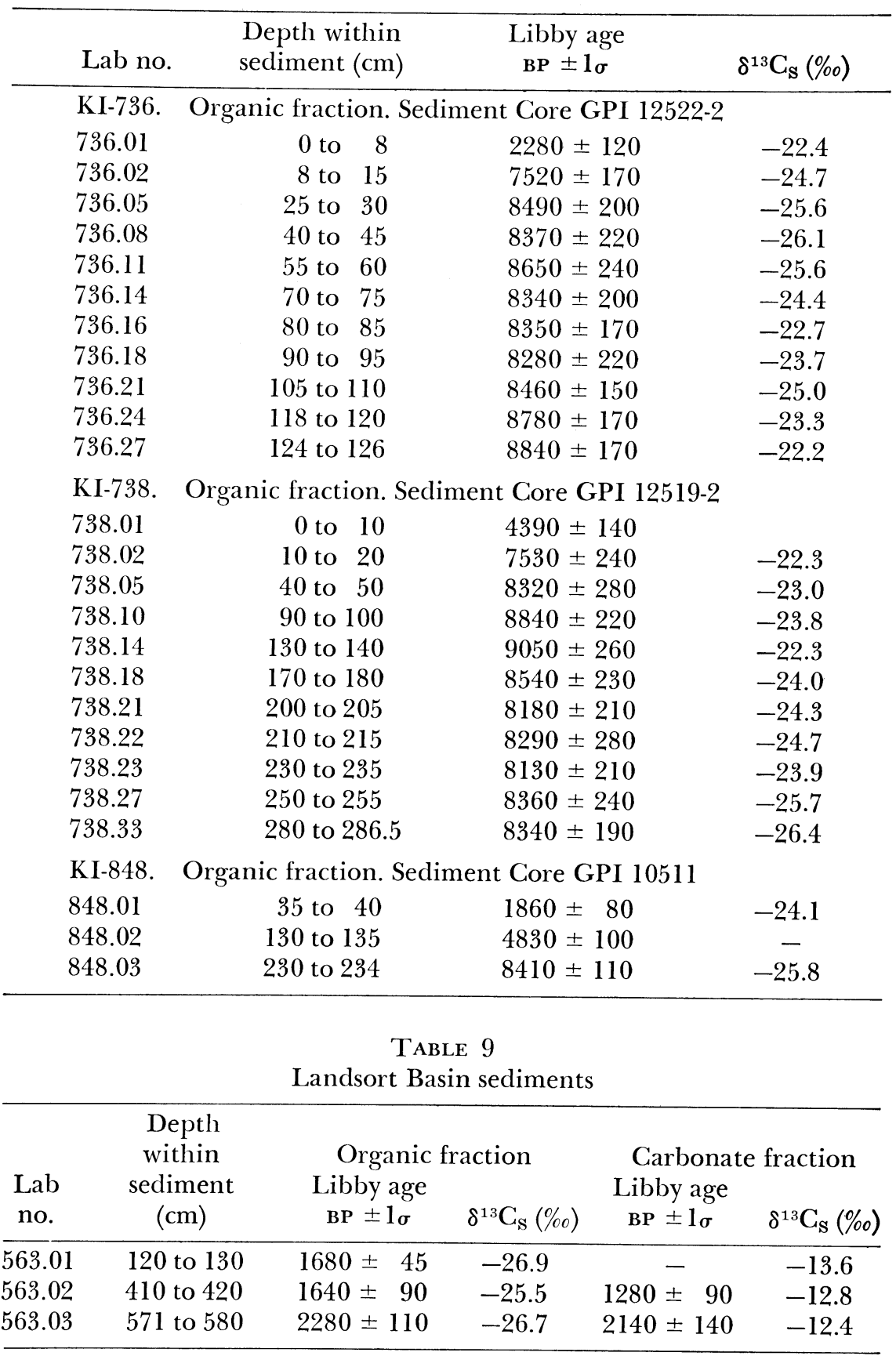




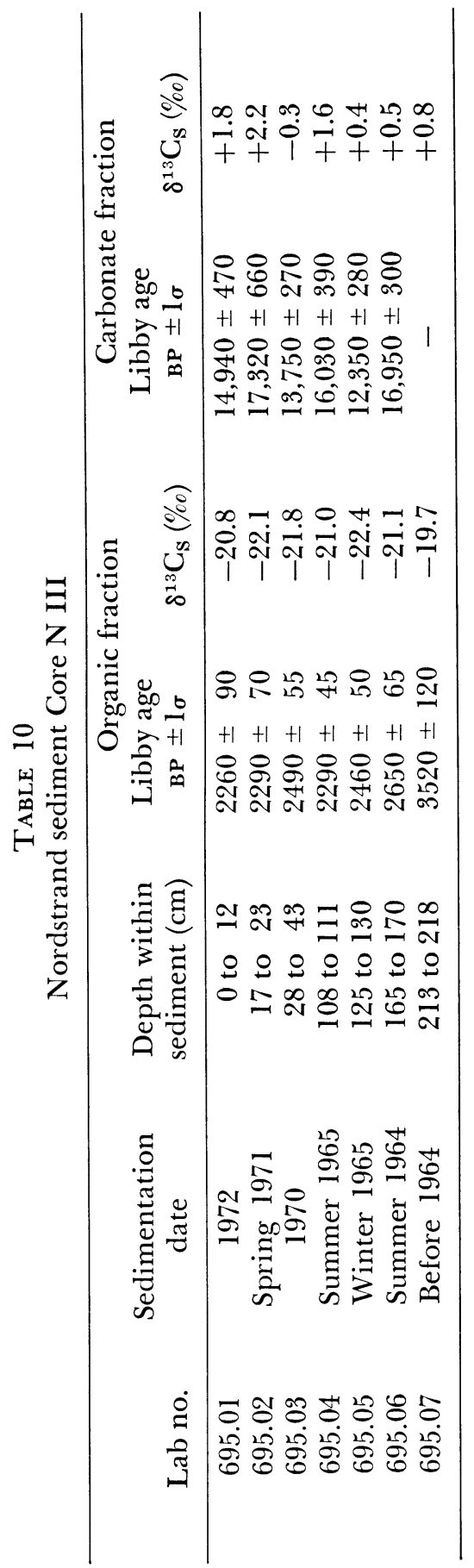




\section{Dredged pit Nordstrand, Suederhafen series}

\section{KI-695. Core N III}

Sediment core, $240 \mathrm{~cm}$ long, coll Nov 1972 by Gerhard Unsöld, Geol Palaeont Inst Univ Kiel at $+70 \mathrm{~cm}$ in foreland of SE Nordstrand $\left(54^{\circ} 28^{\prime}\right.$ $10^{\prime \prime} \mathrm{N}, 8^{\circ} 55^{\prime} 0^{\prime \prime} \mathrm{E}$ ), island in shoals of SE North Sea. Comment (GU): core comes from deepening, ca 70,000 $\mathrm{m}^{2}$ dredged $1962 / 63$, which now is silting up very quickly at several $\mathrm{dm} / \mathrm{yr}$. Winter deposits are laminated, while summer layers are completely disturbed by digging animals. This stratification pattern allows direct determination of sedimentation date (Unsöld, 1974). Organic and carbonate fractions were dated (cf Table 10).

\section{KI-823.001. Nordstrand NF 9}

$$
\begin{gathered}
\mathbf{3 3 4 0} \pm \mathbf{5 0} \\
1390 \mathrm{BC} \\
\delta^{13} C_{S}=-0.8 \%
\end{gathered}
$$

Carbonate fraction of sediment deposited during June/July 1973 in a sediment trap near KI-695. Coll and subm by G Unsöld.

\section{KI-824.001. Nordstrand NST 14. \\ $12,150 \pm 110$ $10,190 \mathrm{BC}$ $\delta^{13} C_{S}=-1.7 \%$}

Carbonate fraction of uppermost sediment, 0 to $2.5 \mathrm{~cm}$ depth, deposited cluring fall storms. Coll Dec 1973 by G Unsöld, near KI-823.

\section{KI-825.001. Nordstrand NST 12}

$13,200 \pm 450$ $10,250 \mathrm{BC}$

$$
\delta^{13} C_{s}=-0.4 \%
$$

Material similar to KI-824. Sediment depth 0 to $3 \mathrm{~cm}$.

General Comment: small activity of recent sediments $(74.5 \%$ for organic

\begin{tabular}{|c|c|c|c|c|}
\hline Lab no. & $\begin{array}{l}\text { Depth within } \\
\text { sediment }(\mathrm{cm})\end{array}$ & $\begin{array}{l}\text { Factor of } \\
\text { dilution }\end{array}$ & $\begin{array}{l}\text { Libby age } \\
\text { BP } \pm 1_{\sigma}\end{array}$ & $\delta^{13} \mathrm{C}_{\mathrm{S}}(\% o)$ \\
\hline \multicolumn{5}{|c|}{ Core LY-II-13A coll in $1201 \mathrm{~m}$ water depth $\left(35^{\circ} 58^{\prime} \mathrm{N}, 7^{\circ} 49^{\prime} \mathrm{W}\right)$. } \\
\hline KI-693 & 63 to 80 & 3.96 & $11,430 \pm 680$ & -24.4 \\
\hline KI-793.02 & 193 to 210 & 6.14 & $\begin{array}{r}14,230 \begin{array}{r}+1560 \\
-1300\end{array}\end{array}$ & -24.9 \\
\hline KI-694 & 425 to 440 & 6.10 & $\begin{array}{r}19,700 \begin{array}{r}+3700 \\
-2500\end{array}\end{array}$ & -24.3 \\
\hline \multicolumn{5}{|c|}{ Core LY-II-13 coll in $1259 \mathrm{~m}$ depth $22 \mathrm{~km} \mathrm{E}$ of $13 \mathrm{~A}\left(35^{\circ} 57^{\prime} \mathrm{N}, 7^{\circ} 34^{\prime} \mathrm{W}\right)$} \\
\hline KI-794.01 & 120 to 145 & 3.59 & $12,710 \pm 680$ & -23.0 \\
\hline KI-794.03 & 335 to 355 & 4.20 & $\begin{array}{r}16,280 \begin{array}{r}+1190 \\
-1040\end{array}\end{array}$ & -24.5 \\
\hline
\end{tabular}
fractions, 10 to $20 \%$ for carbonates compared to ca $120 \%$ recent water

TABLE 11

Gibraltar Street sediments 
activity) indicates important admixture of inactive or at least late glacial material. Smaller activity $(64.5 \%)$ of KI-695.07 might represent prebomb value.

\section{Gibraltar Street sediment}

Two cores coll April 1972 from USNS Lynch by Liselotte Hass, Geol Palaeont Inst Univ Kiel, and D J Stanley, 200km W of Gibraltar St to date variations in flow velocity (Diester-Haass, 1973). Samples contained 25 to $30 \% \mathrm{CaCO}_{3}$ which were removed. Only organic component was measured after dilution with inactive $\mathrm{CO}_{2}$. Comment: mean sedimentation rate is $.50 \mathrm{~mm} / \mathrm{yr}$. Extrapolation to sediment surface gives age 10,000 yr BP.

\section{REFERENCES}

Arntz, W E, 1971, Biomasse und Produktion des Makrobenthos in den tieferen Teilen der Kieler Bucht im Jahre 1968: Kieler Meeresforschungen, v 27, p 36-72.

Diester-Haass, L, 1973, No current-reversal at 10,000 BP in the Strait of Gibraltar: Marine Geology, v 15, p M1-M9.

Erlenkeuser, H, Suess, E, and Willkomm, H, 1974, Industrialization affects heavy metal and carbon isotope concentration in recent Baltic Sea sediments: Geochim et Cosmochim Acta, v 38, p 823-842.

Erlenkeuser, $\mathrm{H}$ and Willkomm, H, 1972, Data processing in ${ }^{14} \mathrm{C}$-measurements. I. Recording and statistical check: Atomkernenergie, v 19, p 123-126. 1973a, Data processing in ${ }^{14} \mathrm{C}$-measurements. II. Age calculation: Atomkernenergie, v 22, p 90-94.

1973 b, University of Kiel radiocarbon measurements VII: Radiocarbon, v 15, p 113-126.

Gulliksen, S and Nydal, R, 1972, Further calculations on the C-14 exchange between the ocean and the atmosphere: 8th internatl conf on radiocarbon dating, Proc, Wellington, New Zealand, p 282-296.

Kühlmorgen-Hille, G, 1962, Die jahreszeitlichen Veränderungen in der Bodenfauna der Kieler Bucht: Doctoral thesis, Kiel Univ.

Mook, W G, 1970, Stable carbon and oxygen isotopes of natural waters in the Netherlands, in: Isotope Hydrology 1970, IAEA, Vienna 1970, p 163-190.

Rafter, $T$ A and Grant-Taylor, $T$ L, 1972, 8th internatl conf on radiocarbon dating, Proc, Wellington, New Zealand.

Samtleben, C, 1973, Groessenverteilungen von Populationen, Totengemeinschaften und Klappensammlungen der Muschel Mytilus edulis L: Meyniana, v 23, p 69-92.

Sarnthein, M and Richter, W, 1974, Submarine experiments on benthic colonization of sediments in the Western Baltic Sea I: technical layout: Marine Biology, v 28, p 159-164.

Seibold E et al, 1971: Marine geology of Kiel Bay, in: Sedimentology of parts of Central Europe. Guidebook, 8th internatl sed cong, Heidelberg, p 209-235.

Unsöld, G, 1974, Jahreslagen und Aufwachsraten in Schlicksedimenten eines künstlichen, gezeitenoffenen Sedimentationsbeckens (Wattgebiet südlich Nordstrand/ Nordfriesland): Meyniana, v 26, p 103-111.

Voss, F, 1970, Der Einfluss des jüngsten Transgressionsablaufes auf die Küstenentwicklung der Geltinger Birck im Nordteil der westlichen Ostsee: Die Küste, v 20, p 101113 .

Wattenberg, H, 1949, Die Salzgehaltsverteilung in der Kieler Bucht und ihre Abhängigkeit von Strom- und Wetterlage: Kieler Meeresforschungen, v 6, p 17-30.

Willkomm, $\mathrm{H}$ and Erlenkeuser, $\mathrm{H}, 1970$, University of Kiel radiocarbon measurements V: Radiocarbon, v 12, p 526-533.

Winn, Kyaw, 1974, Present and postglacial sedimentation in the Great Belt Channel (Western Baltic): Doctoral thesis, Kiel Univ. 